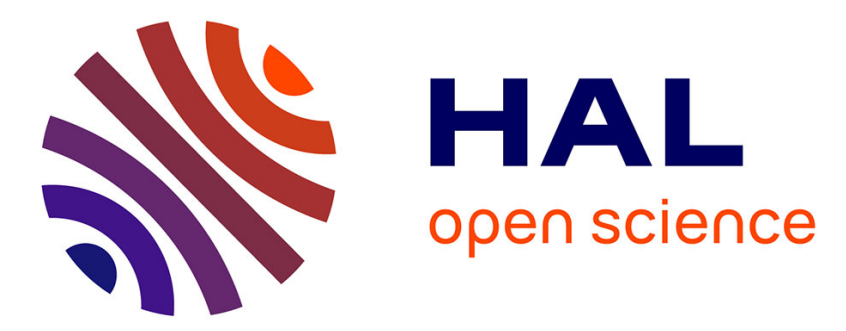

\title{
Energy stable discretization for two-phase porous media flows
}

\author{
Clément Cancès, Flore Nabet
}

\section{To cite this version:}

Clément Cancès, Flore Nabet. Energy stable discretization for two-phase porous media flows. Finite Volumes for Complex Applications IX, Jun 2020, Bergen, Norway. hal-02442233v2

\section{HAL Id: hal-02442233 \\ https://hal.science/hal-02442233v2}

Submitted on 24 Jan 2020

HAL is a multi-disciplinary open access archive for the deposit and dissemination of scientific research documents, whether they are published or not. The documents may come from teaching and research institutions in France or abroad, or from public or private research centers.
L'archive ouverte pluridisciplinaire HAL, est destinée au dépôt et à la diffusion de documents scientifiques de niveau recherche, publiés ou non, émanant des établissements d'enseignement et de recherche français ou étrangers, des laboratoires publics ou privés. 


\title{
Energy stable discretization for two-phase porous media flows
}

\author{
Clément Cancès and Flore Nabet
}

\begin{abstract}
We propose a $\mathbb{P}_{1}$ finite-element scheme with mass-lumping for a model of two incompressible and immiscible phases in a porous media flow. We prove the dissipation of the free energy and the existence of a solution to the nonlinear scheme. We also present numerical simulations to illustrate the behavior of the scheme.
\end{abstract}

Key words: Two-phase porous media flows, energy stable finite-elements

MSC (2010): 65M60, 65M12, 35K65

\section{Immiscible two-phase flows in porous media}

We are interested in the numerical approximations of the equations governing an immiscible incompressible two-phase flow in a porous medium. Let $\Omega \subset \mathbb{R}^{d}$ ( $d=$ 2,3 ) be an open bounded polyhedral subset with Lipschitz boundary condition and let $t_{\mathrm{f}}>0$ be an arbitrary finite time horizon. Then the conservation of the wetting (subscript $\mathrm{w}$ ) and non-wetting phases (subscript $\mathrm{n}$ ) are given by

$$
\phi \partial_{t} s_{\alpha}-\nabla \cdot\left(\eta_{\alpha}\left(s_{\alpha}\right) \Lambda \nabla p_{\alpha}\right)=q_{\alpha}\left(s_{\alpha}\right), \quad \alpha \in\{\mathrm{w}, \mathrm{n}\}
$$

where the unknowns are the phase saturations $s_{\alpha}$, which satisfy

$$
s_{\mathrm{n}}+s_{\mathrm{W}}=1,
$$

Clément Cancès

Inria, Univ. Lille, CNRS, UMR 8524 - Laboratoire Paul Painlevé, F-59000 Lille, France

e-mail: clement.cances@inria.fr

Flore Nabet

CMAP, Ecole polytechnique, CNRS, I.P. Paris, 91128 Palaiseau, France

e-mail: flore.nabet@polytechnique.edu 
and the phase pressures $p_{\alpha}$. The porosity $\phi \in(0,1)$ is given, as well as the intrinsic permeability $\Lambda$, which is assumed to be symmetric and uniformly elliptic. The mobility $\eta_{\alpha}:[0,1] \rightarrow \mathbb{R}$ is assumed to be continuous and strictly increasing, with $\eta_{\alpha}(0)=0$ and $\eta_{\alpha}(s)>0$ if $s>0$. They are extended to the whole $\mathbb{R}$ by $\eta_{\alpha}(s)=0$ if $s<0$ and $\eta_{\alpha}(s)=\eta_{\alpha}(1)$ if $s>1$. The sources $q_{\alpha}$ are such that

$$
q_{\alpha}\left(\mathbf{x}, s_{\alpha}\right)=q_{\mathrm{inj}}(\mathbf{x}) \frac{\eta_{\alpha}\left(c_{\alpha}\right)}{\eta_{\mathrm{w}}\left(c_{\mathrm{w}}\right)+\eta_{\mathrm{n}}\left(c_{\mathrm{n}}\right)}-q_{\mathrm{sink}}(\mathbf{x}) \frac{\eta_{\alpha}\left(s_{\alpha}\right)}{\eta_{\mathrm{w}}\left(s_{\mathrm{w}}\right)+\eta_{\mathrm{n}}\left(s_{\mathrm{n}}\right)},
$$

where $c_{\mathrm{W}} \in(0,1]$ and $c_{\mathrm{n}}=1-c_{\mathrm{W}}$ is the prescribed composition of the injected mixture, and where $q_{\mathrm{inj}}, q_{\mathrm{sink}} \in L^{\infty}(\Omega)$ are nonnegative, bounded, and such that $\int_{\Omega} q_{\mathrm{inj}}=\int_{\Omega} q_{\mathrm{sink}}$. The phase pressures are linked by the capillary pressure relation

$$
p_{\mathrm{n}}-p_{\mathrm{w}}=\gamma\left(s_{\mathrm{n}}\right),
$$

where $\gamma \in L^{1}(0,1)$ is strictly increasing, nonnegative, and blows up as $s_{\mathrm{n}}$ tends to 1. This function is extended for $s<0$ by $\gamma(s)=\gamma(0)+2 s$. We further assume that $s \mapsto \eta_{\mathrm{w}}(1-s) \gamma(s) \in L^{\infty}(0,1)$ and $s \mapsto \eta_{\mathrm{w}}(1-s) \gamma^{\prime}(s) \in L^{1}(0,1)$. These assumptions are satisfied by the usual models of the literature (see for instance [1]). The system is complemented with no-flux boundary conditions and initial conditions $s_{\alpha}^{\text {ini }} \in L^{\infty}(\Omega ;[0,1])$ that are compatible with (2). Note that since $\gamma \in L^{1}(0,1)$, then $\Gamma: s \mapsto \int_{0}^{s} \gamma(a) \mathrm{d} a$ is bounded on $[0,1]$. The phase pressures being only defined up to a constant, we enforce additionally that $\int_{\Omega} p_{\mathrm{n}}=0$.

Multiplying (1) by $p_{\alpha}$, summing over $\alpha \in\{\mathrm{n}, \mathrm{w}\}$, integrating over $\Omega$, and using (2) and (4) yields

$$
\frac{\mathrm{d}}{\mathrm{d} t} \int_{\Omega} \phi \Gamma\left(s_{\mathrm{n}}\right)+\int_{\Omega} \sum_{\alpha \in\{\mathrm{n}, \mathrm{w}\}} \eta_{\alpha}\left(s_{\alpha}\right) \Lambda \nabla p_{\alpha} \cdot \nabla p_{\alpha}=\int_{\Omega} \sum_{\alpha \in\{\mathrm{n}, \mathrm{w}\}} q_{\alpha}\left(s_{\alpha}\right) p_{\alpha} .
$$

Following [6], we define the global pressure $P$ by $P=p_{\mathrm{n}}-r\left(s_{\mathrm{n}}\right)$ with $r: s_{\mathrm{n}} \mapsto$ $\int_{0}^{s_{\mathrm{n}}} \frac{\eta_{\mathrm{w}}(1-a)}{\eta_{\mathrm{n}}(a)+\eta_{\mathrm{w}}(1-a)} \gamma^{\prime}(a) \mathrm{d} a$. The definition of $P$ yields

$$
\sum_{\alpha} \eta_{\alpha}\left(s_{\alpha}\right)\left|\nabla p_{\alpha}\right|^{2}=\left(\eta_{\mathrm{n}}\left(s_{\mathrm{n}}\right)+\eta_{\mathrm{w}}\left(s_{\mathrm{w}}\right)\right)|\nabla P|^{2}+\frac{\eta_{\mathrm{n}}\left(s_{\mathrm{n}}\right) \eta_{\mathrm{w}}\left(s_{\mathrm{w}}\right)}{\eta_{\mathrm{n}}\left(s_{\mathrm{n}}\right)+\eta_{\mathrm{w}}\left(s_{\mathrm{w}}\right)}\left|\nabla \gamma\left(s_{\mathrm{n}}\right)\right|^{2} .
$$

In view of the particular form (3) of the source terms,

$$
\sum_{\alpha \in\{\mathrm{n}, \mathrm{w}\}} q_{\alpha}\left(s_{\alpha}\right) p_{\alpha} \leq\left(q_{\mathrm{inj}}-q_{\mathrm{sink}}\right)\left(P+r\left(s_{\mathrm{n}}\right)\right)+q_{\mathrm{sink}} k\left(s_{\mathrm{n}}\right),
$$

with $k\left(s_{\mathrm{n}}\right)=\frac{\eta_{\mathrm{w}}\left(1-s_{\mathrm{n}}\right)}{\eta_{\mathrm{w}}\left(1-s_{\mathrm{n}}\right)+\eta_{\mathrm{n}}\left(s_{\mathrm{n}}\right)} \gamma\left(s_{\mathrm{n}}\right)$. Since $\eta_{\mathrm{w}}(1-\cdot) \gamma^{\prime} \in L^{1}(0,1)$ and $\eta_{\mathrm{w}}(1-\cdot) \gamma \in$ $L^{\infty}(0,1)$, both $r$ and $k$ are bounded on $(0,1)$. Moreover, the extensions outside $(0,1)$ of $\eta_{\alpha}$ and $\gamma$ ensure that for all $\varepsilon>0$, there exists $C_{\varepsilon}$ such that

$$
|s|+|k(s)|+|r(s)| \leq \varepsilon \Gamma(s)+C_{\varepsilon}, \quad \forall s \in(-\infty, 1) .
$$


Combining (7) with (6) in (5) together with the uniform ellipticity of $\Lambda, \eta_{\mathrm{n}}(s)+$ $\eta_{\mathrm{w}}(1-s) \geq \delta>0$ for all $s$, and (8) we get that

$$
\frac{\mathrm{d}}{\mathrm{d} t} \int_{\Omega} \phi \Gamma\left(s_{\mathrm{n}}\right)+\int_{\Omega}\left(|\nabla P|^{2}+\sum_{\alpha \in\{\mathrm{n}, \mathrm{w}\}} \eta_{\alpha}\left(s_{\alpha}\right)\left|\nabla p_{\alpha}\right|^{2}\right) \leq C .
$$

This estimate is enough to establish the existence of a weak solution. In this paper, our goal is to show that this stability is still encoded in very natural numerical schemes. For the sake of simplicity, we present our analysis in the framework of $\mathbb{P}_{1}$ finite-elements with mass-lumping, but our approach can be extended to a wide family of schemes having the structure highlighted in [3, Section 3]. We show here how to transpose estimate (9) to the discrete setting and to infer the existence of discrete solutions therefrom. A full convergence study will be carried out in a forthcoming contribution. While deeply inspired from [7], the goal of this paper is to exploit more finely the energy estimate which allows to relax some stringent conditions on the anisotropy, on the mesh and the non-linearities presented in [7].

\section{An energy stable finite-element scheme}

We study the problem (1)-(4) using a $\mathbb{P}_{1}$ conforming finite-element scheme with mass-lumping for the space discretization. Let $\mathscr{T}$ be a conforming simplicial discretization of $\Omega$. We denote by $T \in \mathscr{T}$ a simplex, $\mathscr{V}_{\mathscr{T}}$ is the set of all the vertices a and $\mathscr{V}_{T} \subset \mathscr{V}_{\mathscr{T}}$ the set of the $(d+1)$ vertices $\mathbf{a}_{0}, \cdots, \mathbf{a}_{d}$ of the simplex $T$. We also denote by $V_{h}=\left\{u_{h} \in C(\bar{\Omega}):\left.u_{h}\right|_{T}\right.$ is affine for all $\left.T \in \mathscr{T}\right\}$ the usual conforming $\mathbb{P}_{1}$ finite-element space corresponding to the mesh $\mathscr{T}$ and by $\left(\varphi_{\mathbf{a}}\right)_{\mathbf{a} \in \mathscr{V}_{\mathscr{T}}}$ the basis of $V_{h}$. In order to deal with the mass-lumping procedure, for any vertex $\mathbf{a} \in \mathscr{V}_{\mathscr{T}}$, we define the set $\mathfrak{s}_{\mathbf{a}}$, the boundary $\partial_{\mathfrak{s}_{\mathbf{a}}}$ of which being defined by the hyperplanes joining the centers of mass of the simplices, edges (and faces if $d=3$ ) sharing a as a vertex. We can now define the functional space $X_{h}:=\{u \in$ $L^{\infty}(\Omega):\left.u\right|_{\mathfrak{s}_{\mathfrak{a}}}$ is constant for all $\left.\mathbf{a} \in \mathscr{V}_{\mathscr{T}}\right\}$, and the linear mappings $\pi_{X}: C(\bar{\Omega}) \rightarrow X_{h}$ and $\pi_{V}: C(\bar{\Omega}) \rightarrow V_{h}$ by $\pi_{\ell} u(\mathbf{a})=u(\mathbf{a})$, for any $\mathbf{a} \in \mathscr{V}_{\mathscr{T}}$, for any $u \in C(\bar{\Omega}), \ell=X, V$. In order to lighten the notations, for any $u_{h} \in V_{h}$ we write $\pi_{X} u_{h}=\bar{u}_{h}$. We will use the following Poincaré inequality that can be established as in [2]: there exist $C_{1}$, $C_{2}>0$ depending only on the mesh regularity such that for any $u_{h} \in V_{h}$,

$$
\left\|\bar{u}_{h}-\frac{1}{|\Omega|} \int_{\Omega} u_{h}\right\|_{L^{2}(\Omega)} \leq C_{1}\left\|u_{h}-\frac{1}{|\Omega|} \int_{\Omega} u_{h}\right\|_{L^{2}(\Omega)} \leq C_{2}\left\|\nabla u_{h}\right\|_{L^{2}(\Omega)} .
$$

Before detailing the numerical scheme, we have to define the discrete tensor field $\Lambda_{h}: \Omega \rightarrow \mathbb{R}^{d \times d}$ almost everywhere by $\Lambda_{h}(x):=\Lambda_{T}:=\frac{1}{|T|} \int_{T} \Lambda$ if $x \in T$. From there, we define the matrix $\mathbf{A}_{T}:=\left(\alpha_{i, j}^{T}\right)_{1 \leq i, j \leq d} \in \mathbb{R}^{d \times d}$ by 


$$
\alpha_{i, j}^{T}=\alpha_{j, i}^{T}:=\int_{T} \Lambda_{T} \nabla \varphi_{\mathbf{a}_{i}} \cdot \nabla \varphi_{\mathbf{a}_{j}}
$$

and for any $u_{h}, v_{h} \in V_{h}$ one has,

$$
\int_{T} \Lambda_{T} \nabla u_{h} \cdot \nabla v_{h}=\left(\begin{array}{c}
v_{\mathbf{a}_{1}}-v_{\mathbf{a}_{0}} \\
\cdots \\
v_{\mathbf{a}_{d}}-v_{\mathbf{a}_{0}}
\end{array}\right) \cdot \mathbf{A}_{T}\left(\begin{array}{c}
u_{\mathbf{a}_{1}}-u_{\mathbf{a}_{0}} \\
\ldots \\
u_{\mathbf{a}_{d}}-u_{\mathbf{a}_{0}}
\end{array}\right)
$$

Following [4], we can prove that there exists $C_{3}>0$ depending on the regularity of the mesh and on the anisotropy ratio of $\Lambda$ and $C_{4}>0$ depending, in addition, on $d$ such that that for any $T \in \mathscr{T}$ the matrix $\mathbf{A}_{T}$ satisfies

$$
\operatorname{cond}_{2}\left(\mathbf{A}_{T}\right) \leq C_{3} \text { and } \sum_{i=1}^{d}\left(\sum_{j=1}^{d}\left|\alpha_{i, j}^{T}\right|\right)\left(u_{\mathbf{a}_{i}}\right)^{2} \leq C_{4} \mathbf{u} \cdot \mathbf{A}_{T} \mathbf{u}, \forall \mathbf{u}=\left(u_{\mathbf{a}_{i}}\right) \in \mathbb{R}^{d}
$$

We are now in a position to give the numerical scheme using a backward Euler scheme for the time discretization. Let $\left(t^{n}\right)_{n=0, \cdots, N}$ be a partition of the interval $\left[0, t_{\mathrm{f}}\right]$ and for $n=1, \cdots, N$ we denote by $\tau_{n}=t^{n}-t^{n-1}$ the time step. We define the discrete initial data by $s_{\alpha, h}^{0}:=\sum_{\mathbf{a} \in \mathscr{V}_{\mathscr{T}}} s_{\alpha, \mathbf{a}}^{0} \varphi_{\mathbf{a}} \in V_{h}$ with $s_{\alpha, \mathbf{a}}^{0}=\frac{1}{\left|\mathfrak{s}_{\mathbf{a}}\right|} \int_{\mathfrak{s}_{\mathbf{a}}} s_{\alpha}^{\text {ini }}$.

Let $s_{\alpha}^{n-1} \in V_{h}$ be given, we search for $s_{\alpha}^{n}, p_{\alpha}^{n} \in V_{h}$ such that for any $v_{\alpha, h} \in V_{h}$ with $\alpha=(\mathrm{n}, \mathrm{w})$ one has,

$$
\begin{aligned}
\phi \int_{\Omega} \frac{\bar{s}_{\alpha, h}^{n}-\bar{s}_{\alpha, h}^{n-1}}{\tau_{n}} \bar{v}_{\alpha, h}+\int_{\Omega} \eta_{\alpha, h}^{n} \Lambda_{h} \nabla p_{\alpha, h}^{n} \cdot \nabla v_{\alpha, h} & =\int_{\Omega} \bar{q}_{\alpha}\left(\bar{s}_{\alpha, h}^{n}\right) \bar{v}_{\alpha, h}, \\
s_{\mathrm{n}, h}^{n}+s_{\mathrm{w}, h}^{n} & =1 \\
p_{\mathrm{n}, h}^{n}-p_{\mathrm{w}, h}^{n} & =\gamma_{\mathrm{n}, h}^{n}, \\
\int_{\Omega} p_{\mathrm{n}, h}^{n} & =0 .
\end{aligned}
$$

We have denoted by $\eta_{\alpha, h}^{n}=\pi_{V} \eta\left(s_{\alpha, h}^{n}\right), \gamma_{\mathrm{n}, h}^{n}=\pi_{V} \gamma\left(s_{\mathrm{n}, h}^{n}\right)$ and,

$$
\bar{q}_{\alpha}\left(\bar{s}_{\alpha, h}^{n}\right)=\bar{q}_{\text {inj }} \frac{\eta_{\alpha}\left(c_{\alpha}\right)}{\eta_{\mathrm{w}}\left(c_{\mathrm{w}}\right)+\eta_{\mathrm{n}}\left(c_{\mathrm{n}}\right)}-\bar{q}_{\mathrm{sink}} \frac{\eta_{\alpha}\left(\bar{s}_{\alpha, h}^{n}\right)}{\eta_{\mathrm{w}}\left(\bar{s}_{\mathrm{w}, h}^{n}\right)+\eta_{\mathrm{n}}\left(\bar{s}_{\mathrm{n}, h}^{n}\right)} .
$$

Mimicking the continuous case, we define the discrete global pressure and we can obtain the discrete counterpart of (6).

Proposition 1 Let $s_{\alpha, h}^{n}, p_{\alpha, h}^{n} \in V_{h}$ be a solution to the scheme (13). Then there exists $C_{5}>0$ depending on the regularity of the mesh, on the anisotropy ratio of $\Lambda$, on $\delta$ and $d$ such that

$$
\int_{\Omega} \Lambda_{h} \nabla P_{h} \cdot \nabla P_{h} \leq C_{5}\left(\int_{\Omega} \eta_{\mathrm{n}, h}^{n} \Lambda_{h} \nabla p_{\mathrm{n}, h}^{n} \cdot \nabla p_{\mathrm{n}, h}^{n}+\int_{\Omega} \eta_{\mathrm{w}, h}^{n} \Lambda_{h} \nabla p_{\mathrm{w}, h}^{n} \cdot \nabla p_{\mathrm{w}, h}^{n}\right)
$$

where $P_{h}^{n}=p_{\mathrm{n}, h}^{n}-\pi_{V} r\left(s_{\mathrm{n}, h}^{n}\right) \in V_{h}$. 
Proof We define the functions

$$
f_{\mathrm{n}}(s)=\frac{\eta_{\mathrm{n}}(s)}{\eta_{\mathrm{n}}(s)+\eta_{\mathrm{w}}(1-s)} \text { and } f_{\mathrm{w}}(s)=\frac{\eta_{\mathrm{w}}(1-s)}{\eta_{\mathrm{n}}(s)+\eta_{\mathrm{w}}(1-s)}
$$

Then, noting that $f_{\mathrm{n}}+f_{\mathrm{w}}=1$ and using equation (13c), for any $T \in \mathscr{T}$ and for any vertices $\mathbf{a}_{0}, \mathbf{a}_{i} \in \mathscr{V}_{T}$, there exists $s_{i}^{n} \in\left[\min \left(\mathbf{a}_{0}, \mathbf{a}_{i}\right), \max \left(\mathbf{a}_{0}, \mathbf{a}_{i}\right)\right]$ such that,

$$
P_{\mathbf{a}_{0}}^{n}-P_{\mathbf{a}_{i}}^{n}=f_{\mathrm{n}}\left(s_{i}^{n}\right)\left(p_{\mathrm{n}, \mathbf{a}_{0}}^{n}-p_{\mathrm{n}, \mathbf{a}_{i}}^{n}\right)-f_{\mathrm{w}}\left(s_{i}^{n}\right)\left(p_{\mathrm{w}, \mathbf{a}_{0}}^{n}-p_{\mathrm{w}, \mathbf{a}_{i}}^{n}\right) .
$$

Since $\eta_{\alpha}$ is strictly increasing, for any $T \in \mathscr{T}$ with $\mathbf{a}_{0}, \cdots, \mathbf{a}_{d}$ as vertices

$$
\eta_{\alpha, T}^{n}:=\frac{1}{d+1} \sum_{i=0}^{d} \eta_{\alpha}\left(s_{\alpha, \mathbf{a}_{i}}^{n}\right) \geq \frac{1}{d+1} \max _{\mathbf{x} \in T} \eta_{\alpha}(\mathbf{x}) \geq \frac{1}{d+1} \eta_{\alpha}\left(s_{i}^{n}\right)
$$

Thus using that $f_{\mathrm{n}}, f_{\mathrm{w}} \leq 1$ and $\eta_{\mathrm{n}}(s)+\eta_{\mathrm{w}}(1-s) \geq \delta>0$ we obtain,

$$
\frac{\delta}{2(d+1)} \sum_{i=0}^{d}\left|P_{\mathbf{a}_{0}}^{n}-P_{\mathbf{a}_{i}}^{n}\right|^{2} \leq \eta_{\mathrm{n}, T}^{n} \sum_{i=0}^{d}\left|p_{\mathrm{n}, \mathbf{a}_{0}}^{n}-p_{\mathrm{n}, \mathbf{a}_{i}}^{n}\right|^{2}+\eta_{\mathrm{w}, T}^{n} \sum_{i=0}^{d}\left|p_{\mathrm{w}, \mathbf{a}_{0}}^{n}-p_{\mathrm{w}, \mathbf{a}_{i}}^{n}\right|^{2}
$$

Since for any $v_{1}, v_{2}, w$ satisfying $\left|v_{1}\right|^{2}+\left|v_{2}\right|^{2} \geq \operatorname{cond}_{2}\left(\mathbf{A}_{T}\right)|w|^{2}$ one has

$$
v_{1} \cdot \mathbf{A}_{T} v_{1}+v_{2} \cdot \mathbf{A}_{T} v_{2} \geq w \cdot \mathbf{A}_{T} w
$$

we use equality (11) associated with the fact that the condition number of $\mathbf{A}_{T}$ is bounded, cf. (12). Then summing the resulting estimate over $T \in \mathscr{T}$ and noting that the Lagrange vertex-quadrature formula is exact on $\mathbb{P}_{1}$ (see [5, Remark 2.2]) we obtain the claim.

Proposition 2 Let $s_{\alpha, h}^{n-1} \in V_{h}$ be given and $s_{\alpha}^{n}, p_{\alpha}^{n} \in V_{h}$ be a solution to the scheme (13). There exists $C_{6}>0$ depending on the data of the continuous problem but neither on the mesh $\mathscr{T}$ or nor the time step $\tau_{n}$ such that,

$$
\begin{aligned}
\phi \int_{\Omega} \Gamma\left(\bar{s}_{\mathrm{n}, h}^{n}\right)+\tau_{n} \sum_{\alpha \in\{\mathrm{n}, \mathrm{w}\}} \int_{\Omega} \eta_{\alpha, h}^{n} \Lambda_{h} \nabla p_{\alpha, h}^{n} \cdot \nabla p_{\alpha, h}^{n} & +\tau_{n} \int_{\Omega} \nabla P_{h}^{n} \cdot \nabla P_{h}^{n} \\
& \leq C_{6}\left(1+\phi \int_{\Omega} \Gamma\left(\bar{s}_{\mathrm{n}, h}^{n-1}\right)\right) .
\end{aligned}
$$

Proof Let us choose $v_{\alpha, h}=p_{\alpha, h}^{n}$ as test function in equation (13a) and then add the resulting equations. Then, since $\Gamma$ is convex, thanks to relation (13c) we obtain

$$
\begin{aligned}
\phi \int_{\Omega} \Gamma\left(\bar{s}_{\mathrm{n}, h}^{n}\right)+\sum_{\alpha \in\{\mathrm{n}, \mathrm{w}\}} \tau_{n} \int_{\Omega} & \eta_{\alpha, h}^{n} \Lambda_{h} \nabla p_{\alpha, h}^{n} \cdot \nabla p_{\alpha, h}^{n} \\
& \leq \phi \int_{\Omega} \Gamma\left(\bar{s}_{\mathrm{n}, h}^{n-1}\right)+\tau_{n} \int_{\Omega} \sum_{\alpha \in\{\mathrm{n}, \mathrm{w}\}} \bar{q}_{\alpha}\left(\bar{s}_{\alpha, h}^{n}\right) \bar{p}_{\alpha, h}^{n} .
\end{aligned}
$$


As for the continuous case, one has

$$
\sum_{\alpha \in\{\mathrm{n}, \mathrm{w}\}} \bar{q}_{\alpha}\left(\bar{s}_{\alpha, h}^{n}\right) \bar{p}_{\alpha, h}^{n} \leq\left(\bar{q}_{\mathrm{inj}}-\bar{q}_{\mathrm{sink}}\right)\left(\bar{P}_{h}^{n}+r\left(\bar{s}_{\mathrm{n}, h}^{n}\right)\right)+\bar{q}_{\mathrm{sink}} k\left(\bar{s}_{\mathrm{n}, h}^{n}\right) .
$$

Using the definition of the discrete global pressure $P_{h}^{n}$ and equation (13d), combined with the discrete Poincaré inequality (10) and (8) give

$$
\begin{aligned}
\left\|\bar{P}_{h}^{n}\right\|_{L^{1}(\Omega)} & \leq C_{2}|\Omega|^{1 / 2}\left\|\nabla P_{h}^{n}\right\|_{L^{2}(\Omega)}+\int_{\Omega}\left|r\left(\bar{s}_{\mathrm{n}, h}^{n}\right)\right| \\
& \leq \varepsilon\left\|\nabla P_{h}^{n}\right\|_{L^{2}(\Omega)}^{2}+\varepsilon\left\|\Gamma\left(\bar{s}_{\mathrm{n}, h}^{n}\right)\right\|_{L^{1}(\Omega)}+C_{\varepsilon} .
\end{aligned}
$$

Since $q_{\mathrm{inj}}, q_{\mathrm{sink}} \in L^{\infty}(\Omega)$, the use of the above inequality and of (8) in (16) leads to

$$
\int_{\Omega} \sum_{\alpha \in\{\mathrm{n}, \mathrm{w}\}} \bar{q}_{\alpha}\left(\bar{s}_{\alpha, h}^{n}\right) \bar{p}_{\alpha, h}^{n} \leq \varepsilon\left\|\nabla P_{h}^{n}\right\|_{L^{2}(\Omega)}^{2}+\varepsilon\left\|\Gamma\left(\bar{s}_{\mathrm{n}, h}^{n}\right)\right\|_{L^{1}(\Omega)}+C_{\varepsilon}
$$

whatever $\varepsilon>0$. Using (18) together with Proposition 1 in (15) provides the expected bound.

Thanks to equations (13b) and (13c) we see that the saturations and the pressures of the wetting and non-wetting phases are linked. Thus we can choose the pressure of the wetting phase and the capillary pressure as main unknowns. Choosing $v_{\alpha, h}=$ $\varphi_{\mathbf{a}}$ as test functions in equations (13a) we can rewrite the scheme (13) as a nonlinear system of $2 \# \mathscr{V}_{\mathscr{T}}$ algebraic equations $\mathscr{F}^{n}\left(\left(\gamma\left(s_{\mathrm{n}, \mathbf{a}}^{n}\right), p_{\mathrm{w}, \mathbf{a}}^{n}\right)_{\mathbf{a} \in \mathscr{V}_{\mathscr{T}}}\right)=0$. Since $\gamma(1)=$ $+\infty$, the function $\mathscr{F}^{n}$ is continuous but non uniformly continuous. However, we prove in the following lemma that this situation is avoided for a solution to the scheme (13).

Proposition 3 Let $s_{\alpha, h}^{n-1} \in V_{h}$ be such that $\int_{\Omega} \bar{s}_{\mathrm{w}, h}^{n-1} \geq 0$ and $s_{\alpha, \mathrm{h}}^{n}, p_{\alpha, \mathrm{h}}^{n} \in V_{h}$ be a solution the scheme (13). There exists $\sigma_{\tau_{n}, \mathscr{T}}, \varepsilon_{\tau_{n}, \mathscr{T}}>0$ depending on the data of the continuous problem, $\mathscr{T}, \tau_{n}$ and $s_{\mathrm{n}, h}^{n-1}$ such that,

$$
-\sigma_{\tau_{n}, \mathscr{T}} \leq s_{\mathrm{n}, \mathbf{a}}^{n} \leq 1-\varepsilon_{\tau_{n}, \mathscr{T}}, \quad \forall \mathbf{a} \in \mathscr{V}_{\mathscr{T}} .
$$

Proof First of all, thanks to the extension of $\gamma$ for $s<0$, the energy estimate given in Proposition 2 yields $\int_{\Omega}\left(\left(\bar{s}_{\mathrm{n}, h}^{n}\right)^{-}\right)^{2} \leq C^{n-1}$, which provides the lower bound.

Then we prove a bound on the pressure of the non-wetting phase $p_{\mathrm{n}, h}$. Thanks to inequality (17) and the definition of $P_{h}^{n}$ one has

$$
\left\|\bar{p}_{\mathrm{n}, h}^{n}\right\|_{L^{1}(\Omega)} \leq C^{n-1} \Rightarrow\left|p_{\mathrm{n}, \mathbf{a}}^{n}\right| \leq \frac{C^{n-1}}{\left|\mathfrak{s}_{\mathbf{a}}\right|}, \forall \mathbf{a} \in \mathscr{V}_{\mathscr{T}}
$$

Now, let us note that proving the upper bound is equivalent to proving that there exists $\gamma_{\tau_{n}, \mathscr{T}}^{\star}$ such that for any $\mathbf{a} \in \mathscr{V}_{\mathscr{T}}, \gamma\left(s_{\mathrm{n}, \mathbf{a}}\right) \leq \gamma_{\tau_{n}, \mathscr{T}}^{\star}$.

We choose $v_{\mathrm{w}, h}=1$ as test function in equation (13a), then since $q_{\text {inj }}$ is nonnegative, $\eta_{\mathrm{n}}(s)+\eta_{\mathrm{w}}(1-s) \geq \delta>0$ and $c_{\mathrm{w}}>0$ (and so $\eta_{\mathrm{w}}\left(c_{\mathrm{w}}\right)>0$ ), one has 


$$
s_{\mathrm{w}, \mathbf{a}_{\mathrm{i}}}^{n} \geq \frac{1}{|\Omega|} \int_{\Omega} \bar{s}_{\mathrm{w}, h}^{n}>\frac{1}{|\Omega|}\left(\int_{\Omega} \bar{s}_{\mathrm{w}, h}^{n-1}-\frac{\tau_{n}}{\delta}\left\|q_{\operatorname{sink}}\right\|_{L^{\infty}(\Omega)} \int_{\Omega} \eta_{\mathrm{w}}\left(\bar{s}_{\mathrm{n}, h}^{n}\right)\right) .
$$

Note that we proved here by induction that $\int_{\Omega} \bar{s}_{\mathrm{w}, h}^{n} \geq 0$. Since $s \mapsto\left(s+\eta_{\mathrm{w}}(s)\right)^{-1}$ is Lipschitz, there exists $\mathbf{a}_{\mathrm{i}} \in \mathscr{V}_{\mathscr{T}}$ such that $s_{\mathrm{W}, \mathbf{a}_{\mathrm{i}}}^{n}>0$ that is there exists $\mathbf{a}_{\mathrm{i}} \in \mathscr{V}_{\mathscr{T}}$ such that $s_{\mathrm{n}, \mathbf{a}_{\mathrm{i}}}^{n}<1$.

Let $\mathbf{a}_{\mathrm{f}} \in \mathscr{V}_{\mathscr{T}}$ be arbitrary and $\left(\mathbf{a}_{q}\right)_{q=0, \cdots, \ell}$ be a path from $\mathbf{a}_{\mathrm{i}}$ to $\mathbf{a}_{\mathrm{f}}$. Let $q \in$ $\{0, \cdots, \ell-1\}$. Using the property (12) of the matrix $\mathbf{A}_{T}$ and since the quadrature formula is exact on $\mathbb{P}_{1}$, Proposition 2 gives

$$
\sum_{T \in \mathscr{T}} \eta_{\mathrm{w}, T}^{n} \sum_{i=1}^{d}\left(\sum_{j=1}^{d}\left|\alpha_{i, j}^{T}\right|\right)\left(p_{\mathrm{w}, h}^{n}\left(\mathbf{a}_{i}\right)-p_{\mathrm{w}, h}^{n}\left(\mathbf{a}_{0}\right)\right)^{2} \leq \frac{C_{4} C_{6}}{\tau_{n}}\left(1+\phi \int_{\Omega} \Gamma\left(\bar{s}_{\mathrm{n}, h}^{n-1}\right)\right)
$$

We assume by induction that there exists $\varepsilon_{\tau_{n}, \mathscr{T}}>0$ such that $s_{\mathrm{n}, \mathbf{a}_{q}}^{n}<1-\varepsilon_{\tau_{n}, \mathscr{T}}$ that is $s_{\mathrm{w}, \mathbf{a}_{q}}^{n}>\varepsilon_{\tau_{n}, \mathscr{T}}$. Thus, if $T$ is a simplex with $\mathbf{a}_{q}, \mathbf{a}_{q+1}$ as vertices, the definition (14) of $\eta_{\mathrm{w}, T}^{n}$ yields $\eta_{\mathrm{w}, T}^{n} \geq \frac{\eta\left(s_{\mathrm{w}, \mathbf{a}_{q}}^{n}\right)}{d+1} \geq \varepsilon_{\tau_{n}, \mathscr{T}}^{\prime}$. Thanks to equations (13c) and (19) it follows that,

$$
\left|\gamma\left(s_{\mathrm{n}, \mathbf{a}_{q}}^{n}\right)-\gamma\left(s_{\mathrm{n}, \mathbf{a}_{q+1}}^{n}\right)\right|-\left|p_{\mathrm{n}, \mathbf{a}_{q}}^{n}-p_{\mathrm{n}, \mathbf{a}_{q+1}}^{n}\right| \leq C_{\tau_{n}, \mathscr{T}} \quad \Rightarrow \quad \gamma\left(s_{\mathrm{n}, \mathbf{a}_{q+1}}^{n}\right) \leq \gamma_{\tau_{n}, \mathscr{T}}^{\star \star} .
$$

We conclude the proof by induction along the path.

The bound on the saturation associated with the definition (14) on $\eta_{\mathrm{w}, T}^{n}$ yields $\eta_{\mathrm{w}, T}^{n} \geq \eta_{\mathrm{w}}\left(\varepsilon_{\tau_{n}, \mathscr{T}}\right)$. This, combined with the Poincaré inequality (10) and since $\gamma\left(s_{\mathrm{n}, \mathbf{a}}^{n}\right) \leq \gamma\left(1-\varepsilon_{\tau_{n}, \mathscr{T}}\right)$ for any $\mathbf{a} \in \mathscr{V}_{\mathscr{T}}$, allows us to obtain a discrete bound on the pressure.

Proposition 4 There exists $p_{\tau_{n}, \mathscr{T}}^{\star}>0$ depending on the data of the continuous problem, $\mathscr{T}, \tau_{n}$ and $s_{\mathrm{n}, h}^{n-1}$ such that $\int_{\Omega}\left|p_{\mathrm{w}, h}^{n}\right|^{2} \leq p_{\tau_{n}, \mathscr{T}}^{\star}$.

Thanks to the material introduced above, it is possible to prove the existence of a solution to the discrete problem using the topological degree theory.

Theorem 1 (Existence of a solution) Let $s_{\mathrm{n}, h}^{n-1} \in V_{h}$ be given, there exists at least one solution to the scheme (13).

\section{Numerical results}

We present here numerical results obtained with the software FreeFem [8] in the two-dimension case by choosing as main unknowns the saturation of the nonwetting phase and the pressure of the wetting phase. To solve the nonlinear system we use a Newton method with a stopping criteria on the $\ell^{\infty}$-norm between two successive iterations. The computational domain is the unit square $\Omega=] 0,1\left[^{2}\right.$ and the mesh is made up of triangles whose mesh size is approximately equal to 0.028 . The 
final time is $t_{\mathrm{f}}=0.015$ and the time step is constant $\tau_{n}=10^{-3}$. We choose the porosity $\phi=0.3$, the permeability tensor field $\Lambda=\left(\begin{array}{cc}1 & 0 \\ 0 & 100\end{array}\right)$ and $c_{\mathrm{w}}=0.2$. For $s \in[0,1]$ we define the mobility functions by $\eta_{\mathrm{n}}(s)=s^{2}$ and $\eta_{\mathrm{w}}(s)=2 s$, the capillary pressure by $\gamma(s)=\frac{1}{\sqrt{1-s}}$ and the source functions are defined by $q_{\text {inj }}=40.1_{[0,0.2] \times[0.8,1]}$ and $q_{\text {sink }}=40.1_{[0.8,1] \times[0,0.2]}$. We plot in Figure 1 the approximate saturation of the non-wetting phase.

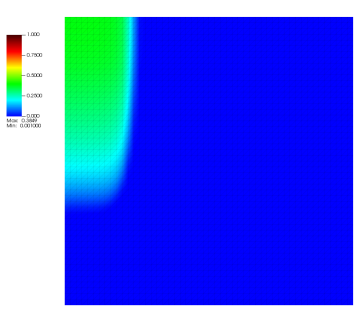

(a) $t=0.002$

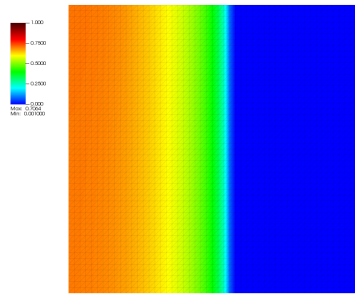

(b) $t=0.01$

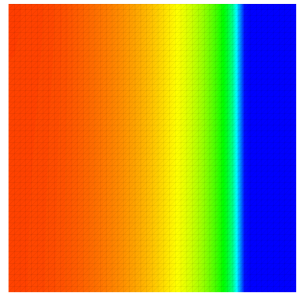

(c) $t=0.015$

Fig. 1: Approximate saturation $s_{\mathrm{n}, h}$ in $\Omega$ for different times $t$.

One observes from the outset of the simulation the influence on the injection well $q_{\text {inj }}$ and of the anisotropy ratio in the longitudinal direction. Moreover we can see that the maximum does not exceed $c_{\mathrm{n}}=0.8$.

Acknowledgements. This work was supported by the French National Research Agency (ANR) through grant ANR-13-JS01-0007-01 (GEOPOR project). Clément Cancès acknowledges the support of Labex CEMPI (ANR-11-LABX-0007).

\section{References}

1. Bear, J., Bachmat, Y.: Introduction to modeling of transport phenomena in porous media. Kluwer Academic Publishers, Dordrecht, The Netherlands (1990)

2. Brenner, K., Masson, R.: Convergence of a vertex centered discretization of two-phase darcy flows on general meshes. Int. J. Finite Vol. 10, 1-37 (2013)

3. Cancès, C.: Energy stable numerical methods for porous media flow type problems. Oil \& Gas Science and Technology-Rev. IFPEN 73, 1-18 (2018)

4. Cancès, C., Guichard, C.: Numerical analysis of a robust free energy diminishing finite volume scheme for parabolic equations with gradient structure. Found. Comput. Math. 17(6), 15251584 (2017)

5. Cancès, C., Nabet, F., Vohralík, M.: Convergence and a posteriori error analysis for energystable finite element approximations of degenerate parabolic equations (2018). HAL: hal01894884

6. Chavent, G., Jaffré, J.: Mathematical Models and Finite Elements for Reservoir Simulation, vol. 17, stud. math. appl. edn. North-Holland, Amsterdam (1986)

7. Eymard, R., Herbin, R., Michel, A.: Mathematical study of a petroleum-engineering scheme. M2AN Math. Model. Numer. Anal. 37(6), 937-972 (2003)

8. Hecht, F.: New development in FreeFem++. J. Numer. Math. 20(3-4), 251-265 (2012) 